\title{
Diagnosing Faulty Transitions in Recommender User Interface Descriptions
}

\author{
Alexander Felfernig \\ Computer Science and Manufacturing, University Klagenfurt \\ A-9020 Klagenfurt, Austria \\ alexander.felfernig@uni-klu.ac.at
}

\begin{abstract}
Complex assortments of products and services offered by online selling platforms require the provision of sales support systems assisting customers in the product selection process. Knowledge-based recommenders are intelligent sales assistance systems which guide online customers through personalized sales dialogs and automatically determine products which conform to their needs and wishes. Such systems have been successfully applied in a number of application domains such as financial services or digital cameras. In this context, the construction of recommender user interfaces is still a challenging task. In many cases faulty models of recommender user interfaces are defined by knowledge engineers and no automated support for debugging such models is available. In this paper we discuss a formal model for defining the intended behaviour of recommender user interfaces and show the application of modelbased diagnosis concepts which allow the automated debugging of those definitions. Experiences show that this approach significantly increases the productivity of recommender user interface development and maintenance.
\end{abstract}

Keywords: knowledge-based recommenders, diagnosis, user interface design.

\section{Introduction}

Simulating the behaviour of human sales experts, recommender systems $[1,4,6,9,12]$ support customers in the product or service selection process. These technologies are of great importance for making product assortments accessible to online customers without technical product domain knowledge. In this paper we concentrate our discussions on knowledge-based recommenders $[1,4]$ which exploit deep knowledge about the product domain in order to determine products or services fitting to the wishes and needs of the customer. Such representations allow the inclusion of automated debugging mechanisms which support the effective development and maintenance of a recommender application. Two basic aspects have to be considered when implementing a knowledge-based recommender application. Firstly, the relevant set of products has to be identified and transformed into a formal representation, i.e. a recommender knowledge base has to be defined. Such a knowledge base consists of a structural description of the provided set of products, a description of the possible set of customer requirements and a set of constraints restricting the possible combinations of 
customer requirements and product properties. Secondly, a model of a recommender process [4] has to be defined which serves as the basis for personalized dialogs. Knowledge bases and process definitions can be automatically (no programming is needed) translated into an executable advisor, where each state of the process definition corresponds to a Web-page in the generated application [4]. In each state a user can articulate his requirements and depending on the given answers the process control determines the following state. In a final state, product recommendations are displayed, e.g. concrete digital cameras or financial services (savings, bonds, etc.). In this paper we focus on a situation where knowledge engineers develop a model of the user interface of a knowledge-based recommender application. The interfaces of such applications are described by a finite number of states, where state transitions are triggered by user requirements represented by a set of variable settings (see Figure 1).

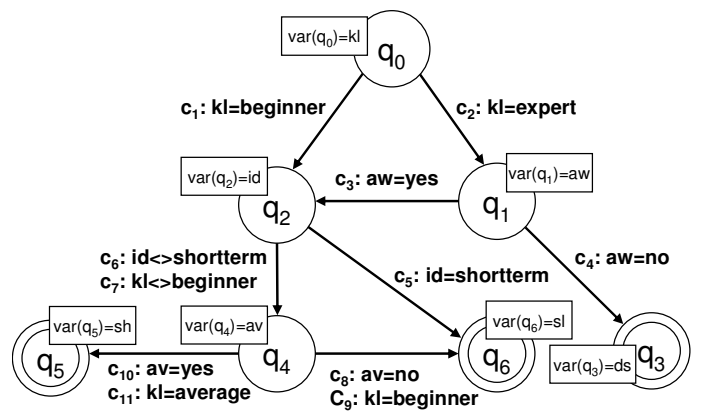

Figure 1: Example (faulty) process definition. $\left\{c_{1}, c_{2}, \ldots, c_{11}\right\}$ are transition conditions between states $\mathrm{q}_{\mathrm{i}}$, $\operatorname{var}\left(\mathrm{q}_{\mathrm{i}}\right)$ represents variables instantiated by the user in state $\mathrm{q}_{\mathrm{i}}$.

Finite state automata [7] can be used for expressing the expected behaviour of a recommender user interface [4]. Typically, customers make decisions by specifying values (requirements) for a subset of a given set of variables. Depending on the answers the automaton changes its state, e.g. an expert ( $\mathrm{kl}=$ expert) who isn't interested in financial services advisory ( $a w=$ no), is forwarded to the state $q_{3}$, where a direct product search can be performed, i.e. different subsets of variables are defined by different paths in the automaton. Note that the design in Figure 1 is faulty, since an expert $(\mathrm{kl}=$ expert) who wants to be guided by a financial advisory process ( $\mathrm{aw}=$ yes) and is interested in long-term investments (id $=$ longterm) and doesn't have any available funds $(\mathrm{av}=\mathrm{no})$ comes to a standstill at the input of availability (the conditions $\left\{c_{2}, c_{9}\right\}$ and $\left\{c_{2}, c_{11}\right\}$ are contradictory). In such a situation, mechanisms are needed allowing an effective identification of the sources of inconsistencies. In the following we show how concepts from model-based diagnosis (MBD) [10] are applied for identifying a minimal set of changes allowing consistency recovery in finite state representations of recommender user interfaces. The presented concepts have been implemented within the scope of the Koba4MS ${ }^{1}$ [4] project, where finite state models are used to specify the intended behaviour of a recommender user interface.

1 Koba4MS is an acronym for Knowledge-based Advisors for Marketing \& Sales (Austrian Research Fund Project 808479). 
The remainder of this paper is organized as follows. In Section 2 we introduce the concept of Predicate-Based Finite State Automata (PFSA), a representation formalism for the design of recommender user interfaces. In Section 3 we discuss our approach to the automated debugging of PFSA-based representations using Model-Based Diagnosis (MBD). In Section 4 we present results from evaluating the presented concepts.

\section{Finite State Models of Recommender User Interfaces}

For the following discussions on the application of model-based diagnosis concepts to the automated debugging of recommender user interface descriptions we introduce a variant of Predicate-Based Finite State Automata (PFSA) [15] (see e.g. Figure 1) which is a natural and compact approach to define state transitions (transitions are defined in terms of domain restrictions of finite domain variables). Note that we restrict our discussions to the case of acyclic automata.

Definition 1 (PFSA): we define a Predicate-based Finite State Automaton (PFSA) to be a 6-tuple $(\mathrm{Q}, \Sigma, \Pi, \mathrm{E}, \mathrm{S}, \mathrm{F})$, where

- $\mathrm{Q}=\left\{\mathrm{q}_{1}, \mathrm{q}_{2}, \ldots, \mathrm{q}_{\mathrm{j}}\right\}$ is a finite set of states, where $\operatorname{var}\left(\mathrm{q}_{\mathrm{i}}\right)=\mathrm{x}_{\mathrm{i}}$ is a finite domain variable assigned to $\mathrm{q}_{\mathrm{i}}, \operatorname{prec}\left(\mathrm{q}_{\mathrm{i}}\right)=\left\{\phi_{1}, \phi_{2}, \ldots, \phi_{\mathrm{m}}\right\}$ is the set of preconditions of $\mathrm{q}_{\mathrm{i}}$ $\left(\phi_{\mathrm{k}}=\left\{\mathrm{c}_{\mathrm{r}}, \mathrm{c}_{\mathrm{s}}, \ldots, \mathrm{c}_{\mathrm{t}}\right\} \subseteq \Pi\right), \operatorname{postc}\left(\mathrm{q}_{\mathrm{i}}\right)=\left\{\psi_{1}, \psi_{2}, \ldots, \psi_{\mathrm{n}}\right\}$ is the set of postconditions of $\mathrm{q}_{\mathrm{i}}\left(\psi_{\mathrm{l}}=\left\{\mathrm{c}_{\mathrm{u}}, \mathrm{c}_{\mathrm{v}}, \ldots, \mathrm{c}_{\mathrm{w}}\right\} \subseteq \Pi\right)$, and $\operatorname{dom}\left(\mathrm{x}_{\mathrm{i}}\right)=\left\{\mathrm{x}_{\mathrm{i}}=\mathrm{d}_{\mathrm{i} 1}, \mathrm{x}_{\mathrm{i}}=\mathrm{d}_{\mathrm{i} 2}, \ldots, \mathrm{x}_{\mathrm{i}}=\mathrm{d}_{\mathrm{ip}}\right\}$ denotes the set of possible assignments of $x_{i}$, i.e. the domain of $x_{i}$.

- $\Sigma=\left\{\mathrm{x}_{\mathrm{i}}=\mathrm{d}_{\mathrm{ij}} \mid \mathrm{x}_{\mathrm{i}}=\operatorname{var}\left(\mathrm{q}_{\mathrm{i}}\right), \mathrm{x}_{\mathrm{i}}=\mathrm{d}_{\mathrm{ij}} \in \operatorname{dom}\left(\mathrm{x}_{\mathrm{i}}\right)\right\}$ is a finite set of variable assignments (input symbols), the input alphabet.

- $\Pi=\left\{c_{1}, c_{2}, \ldots, c_{q}\right\}$ is a set of constraints (transition conditions) restricting the set of words accepted by the PFSA.

- $\quad$ E is a finite set of transitions $\subseteq \mathrm{Q} \times \Pi \times \mathrm{Q}$.

- $\mathrm{S} \subseteq \mathrm{Q}$ is a set of start states.

- $\mathrm{F} \subseteq \mathrm{Q}$ is a set of final states. $\square$

Preconditions of a state $\mathrm{q}_{\mathrm{i}}\left(\operatorname{prec}\left(\mathrm{q}_{\mathrm{i}}\right)=\left\{\phi_{1}, \phi_{2}, \ldots, \phi_{\mathrm{m}}\right\}\right)$ can be automatically derived from the reachability tree of a Predicate-based Finite State Automaton. Figure 2 depicts the reachability tree for the PFSA of Figure 1. The state $q_{2}$ is accessed twice in the reachability tree. The corresponding preconditions $\left(\operatorname{prec}\left(\mathrm{q}_{2}\right)\right)$ are derived from the transition conditions of the paths leading to $\mathrm{q}_{2}$, i.e. $\left\{\left\{\mathrm{c}_{1}\right\},\left\{\mathrm{c}_{2}, \mathrm{c}_{3}\right\}\right\}$. Similarly, $\operatorname{postc}\left(\mathrm{q}_{\mathrm{i}}\right)$ is the set of postconditions of the state $\mathrm{q}_{i}$ which are as well derived from the reachability tree, e.g. the state $\mathrm{q}_{4}$ has two postconditions, namely $\left\{\left\{\mathrm{c}_{8}, \mathrm{c}_{9}\right\},\left\{\mathrm{c}_{10}, \mathrm{c}_{11}\right\}\right\}$. Example 1 includes a PFSA describing the behaviour of a financial services advisory dialog (the graphical representation is contained in Figure 1). States in the PFSA represent questions which can be posed to customers during an advisory session. Subsequent states in the PFSA are determined depending on the answers given by a customer. We denote the set of input sequences leading to a final state of a PFSA as the accepted language. 
In the context of user interface development for recommender applications, supporting concepts are needed which allow the effective identification of faulty transition conditions, e.g. if a sequence of user inputs reaches state $q_{i}$, there must exist an extension of this input sequence to a final state of the PFSA. Path expressions are the basis for expressing well-formedness properties on process definitions (see Definition 2).

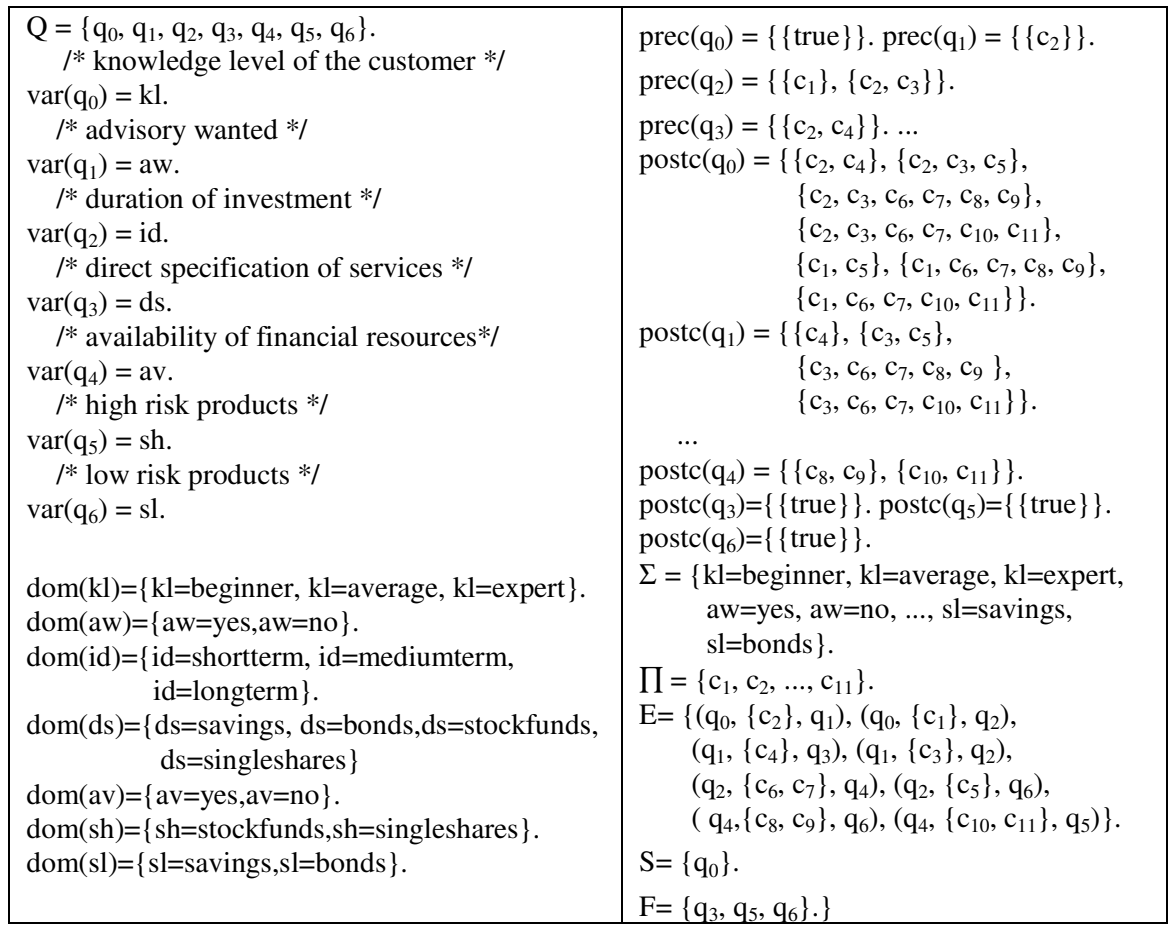

Example 1: Predicate-based Finite State Automaton (PFSA).

Definition 2 (consistent path): Let $\mathrm{p}=\left[\left(\mathrm{q}_{1}, \mathrm{C}_{1}, \mathrm{q}_{2}\right),\left(\mathrm{q}_{2}, \mathrm{C}_{2}, \mathrm{q}_{3}\right), \ldots, \quad\left(\mathrm{q}_{\mathrm{i}-1}, \mathrm{C}_{\mathrm{i}-1}, \mathrm{q}_{\mathrm{i}}\right)\right]$ $\left(\left(\mathrm{q}_{\alpha}, \mathrm{C}_{\alpha}, \mathrm{q}_{\beta}\right) \in \mathrm{E}\right)$ be a path from a state $\mathrm{q}_{1} \in \mathrm{S}$ to a state $\mathrm{q}_{\mathrm{i}} \in \mathrm{Q}$. $\mathrm{p}$ is consistent (consistent(p)) iff $\cup \mathrm{C}_{\mathrm{j}}$ is satisfiable.

Using the notion of a consistent path we can now introduce the following set of wellformedness rules specifying structural properties of a PFSA. These rules are relevant for building knowledge-based recommender applications. Due to the generality of the presented diagnosis approach, we can introduce further well-formedness properties for domain-specific purposes.

A consistent path in a PFSA leading to a state $\mathrm{q}_{\mathrm{i}}$ must have a direct postcondition, i.e., $\left(\mathrm{q}_{\mathrm{i}}, \mathrm{C}_{\mathrm{i}}, \mathrm{q}_{\mathrm{j}}\right)$ propagating the path (i.e. each consistent path must be extensible). 
Definition 3 (extensible path): Let $\mathrm{p}=\left[\left(\mathrm{q}_{1}, \mathrm{C}_{1}, \mathrm{q}_{2}\right),\left(\mathrm{q}_{2}, \mathrm{C}_{2}, \mathrm{q}_{3}\right), \ldots,\left(\mathrm{q}_{\mathrm{i}-1}, \mathrm{C}_{\mathrm{i}-1}, \mathrm{q}_{\mathrm{i}}\right)\right]$ be a path from a state $\mathrm{q}_{1} \in \mathrm{S}$ to a state $\mathrm{q}_{\mathrm{i}} \in \mathrm{Q}-\mathrm{F}$. p is extensible (extensible(p)) iff $\exists$ $\left(\mathrm{q}_{\mathrm{i}}, \mathrm{C}_{\mathrm{i}}, \mathrm{q}_{\mathrm{i}+1}\right): \mathrm{C}_{1} \cup \mathrm{C}_{2} \cup \ldots \cup \mathrm{C}_{\mathrm{i}-1} \cup \mathrm{C}_{\mathrm{i}}$ is satisfiable. $\square$

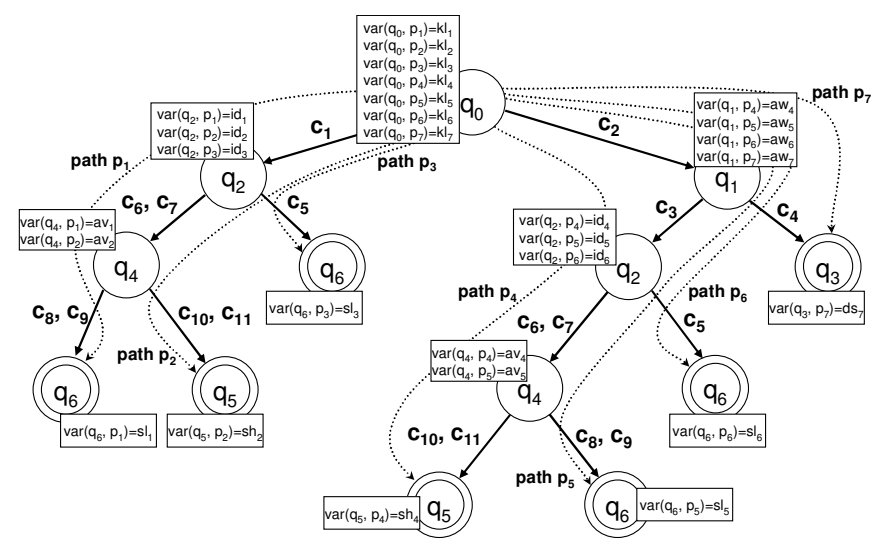

Figure 2: Reachability tree of a PFSA. The tree represents the expansion of the PFSA shown in Figure 1. \{p1, p2, ..., p7 $\}$ are the possible paths of the PFSA. For each path $\mathrm{p}_{\mathrm{i}}$ the corresponding path variables are shown.

A state $\mathrm{q}_{\mathrm{i}}$ is a decision point for determining the subsequent state. The selection of subsequent states depends on the definition of the direct postconditions for $\mathrm{q}_{\mathrm{i}}$, where each postcondition has to be unique for determining the subsequent state. A state $q_{i}$ is deterministic if each of its postconditions is unique for determining subsequent states.

Definition 4 (deterministic state): Let $\mathrm{p}=\left[\left(\mathrm{q}_{1}, \mathrm{C}_{1}, \mathrm{q}_{2}\right),\left(\mathrm{q}_{2}, \mathrm{C}_{2}, \mathrm{q}_{3}\right), \ldots,\left(\mathrm{q}_{\mathrm{i}-1}, \mathrm{C}_{\mathrm{i}-1}, \mathrm{q}_{\mathrm{i}}\right)\right]$ be a path from a state $\mathrm{q}_{1} \in \mathrm{S}$ to a state $\mathrm{q}_{\mathrm{i}} \in \mathrm{Q}-\mathrm{F}$. A state $\left(\mathrm{q}_{\mathrm{i}}\right)$ is deterministic $\left(\mathrm{q}_{\mathrm{i}}\right)$ iff $\forall\left(\mathrm{q}_{\mathrm{i}}, \mathrm{C}_{\mathrm{i} 1}, \mathrm{q}_{\mathrm{j}}\right),\left(\mathrm{q}_{\mathrm{i}}, \mathrm{C}_{\mathrm{i} 2}, \mathrm{q}_{\mathrm{k}}\right) \in \mathrm{E}: \mathrm{C}_{1} \cup \mathrm{C}_{2} \cup \ldots \cup \mathrm{C}_{\mathrm{i}-1} \cup \mathrm{C}_{\mathrm{i}} \cup \mathrm{C}_{\mathrm{i} 2}$ is contradictory $\left(\mathrm{C}_{\mathrm{i} 1} \neq \mathrm{C}_{\mathrm{i} 2}\right)$.

All transitions of a PFSA should be accessible, i.e. for each transition there exists at least one corresponding path including this transition.

Definition 5 (accessible transition): A transition $t=\left(\mathrm{q}_{\mathrm{i}}, \mathrm{C}_{\mathrm{i}}, \mathrm{q}_{\mathrm{i}+1}\right)$ (postcondition of state $\left.\mathrm{q}_{\mathrm{i}}\right)$ is accessible $(\operatorname{accessible}(\mathrm{t}))$ iff there exists a path $\mathrm{p}=\left[\left(\mathrm{q}_{1}, \mathrm{C}_{1}, \mathrm{q}_{2}\right),\left(\mathrm{q}_{2}, \mathrm{C}_{2}, \mathrm{q}_{3}\right), \ldots\right.$, $\left.\left(\mathrm{q}_{\mathrm{i}-1}, \mathrm{C}_{\mathrm{i}-1}, \mathrm{q}_{\mathrm{i}}\right)\right]\left(\mathrm{q}_{1} \in \mathrm{S}\right): \mathrm{C}_{1} \cup \mathrm{C}_{2} \cup \ldots \cup \mathrm{C}_{\mathrm{i}-1} \cup \mathrm{C}_{\mathrm{i}}$ is satisfiable. $\square$

A PFSA is well-formed, if the set of defined well-formedness rules is fulfilled.

Definition 6 (well-formed PFSA): A PFSA is well-formed iff

- each consistent path $\mathrm{p}=\left[\left(\mathrm{q}_{1}, \mathrm{C}_{1}, \mathrm{q}_{2}\right),\left(\mathrm{q}_{2}, \mathrm{C}_{2}, \mathrm{q}_{3}\right), \ldots,\left(\mathrm{q}_{\mathrm{i}-1}, \mathrm{C}_{\mathrm{i}-1}, \mathrm{q}_{\mathrm{i}}\right)\right]\left(\mathrm{q}_{1} \in \mathrm{S}, \mathrm{q}_{\mathrm{i}} \in \mathrm{Q}\right.$ $\mathrm{F})$ is extensible to a consistent path p' $=\left[\left(\mathrm{q}_{1}, \mathrm{C}_{1}, \mathrm{q}_{2}\right),\left(\mathrm{q}_{2}, \mathrm{C}_{2}, \mathrm{q}_{3}\right), \ldots,\left(\mathrm{q}_{\mathrm{i}-1}, \mathrm{C}_{\mathrm{i}-1}, \mathrm{q}_{\mathrm{i}}\right)\right.$, $\left.\left(\mathrm{q}_{\mathrm{i}}, \mathrm{C}_{\mathrm{i}}, \mathrm{q}_{\mathrm{j}}\right)\right]$.

- $\forall \mathrm{q}_{\mathrm{k}} \in \mathrm{Q}:$ deterministic $\left(\mathrm{q}_{\mathrm{k}}\right)$.

- $\forall \mathrm{t}=\left(\mathrm{q}_{\mathrm{k}}, \mathrm{C}_{\mathrm{k}}, \mathrm{q}_{\mathrm{l}}\right) \in \mathrm{E}: \operatorname{accessible}(\mathrm{t})$. $\square$ 


\section{Diagnosing Faulty Transitions in User Interface Descriptions}

Faulty transition conditions should be identified as soon as possible. Consequently, we need support in the repair of not well-formed PFSAs by an automated identification of minimal sets of transition conditions responsible for the faulty behaviour. We apply model-based diagnosis (MBD) [10] by introducing the concept of a PFSA Diagnosis Problem and a corresponding PFSA Diagnosis. Our approach starts with the description of a system (SD) which is in our case the description of the structure and the intended behaviour of the PFSA (well-formedness rules discussed in Section 2). If the observable behaviour of the system conflicts with its intended behaviour (some of the well-formedness rules are violated), the task of the diagnosis algorithm is it to determine components (in our case transition conditions) which, when assumed to functioning abnormally, explain the discrepancy between the actual and the intended system behaviour. In order to apply MBD concepts, we transform a PFSA definition into a corresponding constraint-based representation [14] consisting of restrictions representing well-formedness properties and transitions of the PFSA. A calculated diagnosis represents a minimal set of transitions which are responsible for the faulty behaviour of the automaton, i.e. are inconsistent with the defined well-formedness properties (see Section 2). Note that there can be different diagnoses providing an explanation for the faulty behaviour of a PFSA. We define a Predicate-based Finite State Automaton Diagnosis Problem (PFSA Diagnosis Problem) as follows.

Definition 7 (PFSA Diagnosis Problem): A PFSA Diagnosis Problem is represented by a tuple (SD, TRANS), where SD $=$ STAT $\cup$ WF. STAT is the structural description of a PFSA represented by a set of finite domain variables. WF is the intended behaviour of a PFSA which is represented by a set of constraints on STAT. Finally, TRANS represents a set of transition conditions.

The basic elements of a PFSA Diagnosis Problem, i.e., STAT, WF, TRANS, define a corresponding Constraint Satisfaction Problem (CSP) [14]. Solutions for this CSP cover all interaction paths, i.e., accepted runs of the PFSA. In this context, STAT represents a set of finite domain variables related to paths of the reachability tree (the variables of our example PFSA are depicted in Figure 2 and Example 2), e.g. $\left\{\mathrm{kl}_{1}, \mathrm{id}_{1}\right.$, $\left.\mathrm{av}_{1}, \mathrm{sl}_{1}\right\}$ are variables corresponding to the path $\mathrm{p}_{1}$. Note that the projection of all solutions for the CSP (STAT, WF, TRANS) to e.g. the variables $\left\{\mathrm{kl}_{1}, \mathrm{id}_{1}, \mathrm{av}_{1}, \mathrm{sl}_{1}\right\}$ represents input sequences accepted by path $\mathrm{p}_{1}$.

Example 2 (STAT): STAT $=\left\{\mathrm{kl}_{1}, \mathrm{id}_{1}, \mathrm{av}_{1}, \mathrm{sl}_{1}, \mathrm{kl}_{2}, \mathrm{id}_{2}, \mathrm{av}_{2}, \mathrm{sh}_{2}, \mathrm{kl}_{3}, \mathrm{id}_{3}, \mathrm{sl}_{3}, \mathrm{kl}_{4}, \mathrm{aw}_{4}\right.$, $\left.\mathrm{id}_{4}, \mathrm{av}_{4}, \mathrm{sh}_{4}, \mathrm{kl}_{5}, \mathrm{aw}_{5}, \mathrm{id}_{5}, \mathrm{av}_{5}, \mathrm{sl}_{5}, \mathrm{kl}_{6}, \mathrm{aw}_{6}, \mathrm{id}_{6}, \mathrm{sl}_{6}, \mathrm{kl}_{7}, \mathrm{aw}_{7}, \mathrm{ds}_{7}\right\} \square$

The construction of well-formedness rules (Definitions 3-5) $\mathrm{WF}=\mathrm{WF}_{\text {extensibil- }}$ ity $\cup \mathrm{WF}_{\text {accessibility }} \cup \mathrm{WF}_{\text {determinism }}$ is now exemplified by the accessibility rule related to the transition $\left(\mathrm{q}_{2},\left\{\mathrm{c}_{6}, \mathrm{c}_{7}\right\}, \mathrm{q}_{4}\right)$ of our example PFSA (see Figure 1).

Example 3 (well-formedness rules for accessibility): $\mathrm{WF}_{\text {accessibility }}\left(\mathrm{q}_{2},\left\{\mathrm{c}_{6}, \mathrm{c}_{7}\right\}, \mathrm{q}_{4}\right)=$

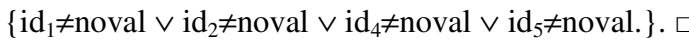


The transition $\left(\mathrm{q}_{2},\left\{\mathrm{c}_{6}, \mathrm{c}_{7}\right\}, \mathrm{q}_{4}\right)$ must be accessible for at least one of the paths $\mathrm{p}_{1}, \mathrm{p}_{2}$, $\mathrm{p}_{4}, \mathrm{p}_{5}$, i.e. at least one of the variables $\mathrm{id}_{1}, \mathrm{id}_{2}, \mathrm{id}_{4}, \mathrm{id}_{5}$ must have a value $\neq$ noval in each solution for the CSP defined by (STAT, WF, TRANS). Since in our case a reachability tree (see e.g. Figure 2) represents the complete expansion of a corresponding PFSA, not all the paths are necessarily consistent. If a path of the reachability tree represents such an illegal trajectory, i.e. no consistent value assignment exists for the corresponding variables, all variables of this path have the assignment noval. This is assured by meta-constraints defined for each path in the reachability tree, e.g. for path $\mathrm{p}_{1}: \mathrm{kl}_{1} \neq$ noval $\wedge \mathrm{id}_{1} \neq$ noval $\wedge \mathrm{av}_{1} \neq$ noval $\wedge \mathrm{sl}_{1} \neq$ noval $\vee \mathrm{kl}_{1}=$ noval $\wedge \mathrm{id}_{1}=$ noval $\wedge \mathrm{av}_{1}=$ noval $\wedge \mathrm{sl}_{1}=$ noval. An example for the definition of a transition condition (TRANS) is the following (see Example 4). We represent the transition condition $\mathrm{c}_{1}$ of our example PFSA.

Example 4 (TRANS for PFSA): TRANS $=\left\{\mathrm{c}_{1}:\left(\mathrm{kl}_{1}=\right.\right.$ beginner $\vee \mathrm{kl}_{1}=$ noval $) \wedge\left(\mathrm{kl}_{2}=\right.$ beginner $\vee \mathrm{kl}_{2}=$ noval $) \wedge\left(\mathrm{kl}_{3}=\right.$ beginner $\vee \mathrm{kl}_{3}=$ noval $\left.) . ..\right\}$. $\square$

The condition generated in Example 4 contains those variables belonging to paths including $\left(\mathrm{q}_{0},\left\{\mathrm{c}_{1}\right\}, \mathrm{q}_{2}\right)$, i.e. $\left\{\mathrm{kl}_{1}, \mathrm{kl}_{2}, \mathrm{kl}_{3}\right\}$ which belong to the paths $\left\{\mathrm{p}_{1}, \mathrm{p}_{2}, \mathrm{p}_{3}\right\}$. For the CSP (STAT, WF, TRANS) the possible values of $\left\{\mathrm{kl}_{1}, \mathrm{kl}_{2}, \mathrm{kl}_{3}\right\}$ are defined by $\mathrm{c}_{1}$, i.e. the value can be either beginner or noval (in the case that the corresponding path is an illegal trajectory). Given a specification of (SD, TRANS), a PFSA Diagnosis is defined as follows.

Definition 8 (PFSA Diagnosis): A PFSA Diagnosis for a PFSA Diagnosis Problem (SD, TRANS) is a set $\mathrm{S} \subseteq$ TRANS s.t. SD $\cup$ TRANS $-\mathrm{S}$ consistent. $\square$

A diagnosis exists under the assumption that STATS $\cup$ WF is consistent [2]. The calculation of diagnoses is based on the concept of minimal conflict sets.

Definition 9 (Conflict Set): a Conflict Set (CS) for (SD, TRANS) is a set $\left\{c_{1}, c_{2}, \ldots\right.$, $\left.\mathrm{c}_{\mathrm{n}}\right\} \subseteq$ TRANS, s.t. $\left\{\mathrm{c}_{1}, \mathrm{c}_{2}, \ldots, \mathrm{c}_{\mathrm{n}}\right\} \cup \mathrm{SD}$ is inconsistent. CS is minimal iff $\neg \exists \mathrm{CS}^{`} \subset \mathrm{CS}$ : conflict set (CS'). $\square$

Algorithm 1 sketches the calculation of diagnoses for a given PFSA.

Algorithm 1: PFSA-Diagnosis (SD, TRANS):

(a) Generate a pruned HSDAG $\mathrm{T}$ for the collection of conflict sets induced by transitions of TRANS in breadth-first manner (we generate diagnoses in order of their cardinality). With every theorem prover (TP) call at a node $n$ of $\mathrm{T}$ the consistency of (TRANS - H(n) $\cup \mathrm{SD}$ ) is checked. If there exists an inconsistency, a conflict set CS is returned, otherwise ok is returned. If (TRANS - H(n) $\cup$ SD) is consistent, a corresponding diagnosis $\mathrm{H}(\mathrm{n})$ is found.

(b) Return $\{\mathrm{H}(\mathrm{n}) \mid \mathrm{n}$ is a node of $\mathrm{T}$ labeled with ok $\}$.

The labelling of the search tree (Hitting Set Directed Acyclic Graph - HSDAG) is based on the labelling of the HSDAG presented in [10]. A node $n$ is labelled by a corresponding conflict set CS(n). The set of edge labels from the root to node $n$ is 
denoted to as $\mathrm{H}(n)$ which represents a corresponding diagnosis (in the case that $n$ is a leaf node). Following our example, the conflict sets which are determined by subsequent TP calls are $\left\{\mathrm{c}_{2}, \mathrm{c}_{11}\right\},\left\{\mathrm{c}_{7}, \mathrm{c}_{9}\right\},\left\{\mathrm{c}_{2}, \mathrm{c}_{9}\right\}$, and $\left\{\mathrm{c}_{1}, \mathrm{c}_{9}\right\}$. One diagnosis for our PFSA is $\left\{c_{2}, c_{9}\right\}$, i.e., at least $\left\{c_{2}, c_{9}\right\}$ have to be changed in order to restore consistency of a given PFSA definition.

\section{Evaluation}

The presented approach to introduce Model-Based Diagnosis (MBD) [10] into the development of knowledge-based recommenders does not change the structure of the user interface supporting the design of recommender processes, but rather complements the existing knowledge acquisition component [4] with concepts supporting the detection of faulty areas in a given PFSA definition. This approach is not restricted to knowledge-based recommendation, but is generally applicable to domains, where a user interface is explicitly defined in terms of a finite state model (in the form of a PFSA). A performance evaluation [2] shows the applicability of the presented algorithm in (interactive) real-world settings. Typically, recommender process definitions consist of about 15-40 states - a size which makes the application of the presented debugging concepts meaningful. In many cases there exist a number of alternative diagnoses explaining the sources of inconsistencies in a given process definition. In this context we will include additional ranking mechanisms for diagnoses which take into account the probability of a transition condition to be faulty (e.g. the probability of a transition condition to be faulty is higher if there exists a large number of incoming paths to the related state). We have compared development efforts related to projects without debugging support for process definitions and with a corresponding debugging support. Our development environment has a logging functionality which stores each activity in the recommender design environment (e.g. change/edit a transition condition, etc.). The projects investigated have a similar structure w.r.t. number of product properties, customer properties, constraints, number of states in the PFSA etc. The observation of time efforts shows that the usage of the debugging concepts can significantly reduce efforts related to the development and maintenance of recommender user interfaces. In order to empirically show the direct positive influence of debugging techniques we have conducted an experiment with ninety-seven participating students all having experiences in developing knowledge-based recommender applications. In this experiment participants were interacting with a web-based application providing the basic functionality of our debugging environment. The participants had to solve the task of identifying a minimal set of constraints in given recommender process definitions which should be changed in order to make the process definition consistent with the well-formedness properties presented in this paper. The participants had to propose a set of repair actions which make the process definition consistent with the well-formedness rules. The hypothesis of the experiment was that automated debugging support leads to effort reductions in the detection and repair of faulty transition conditions. The result of the experiment was a significant decrease of time efforts due to the application of the presented debugging concepts (on an average 
of $36 \%$ - time reductions depend on the number of faulty transitions conditions in the process definition). A detailed analysis of the consequences of applying automated debugging concepts in recommender application development is currently ongoing and is in the line of our research focus to improve the effectiveness of recommender application development processes.

\section{Related Work}

The increasing size and complexity of configuration knowledge bases motivated the application of model-based diagnosis (MBD) [10] in knowledge-based systems development [3]. Similar motivations led to the application of MBD in technical domains such as the development of hardware designs [5], onboard diagnosis for automotive systems [11] and in software development [8]. A value-based model of program execution is introduced in a.o. [8], which is an extension of the approach of debugging imperative languages [5]. Using MBD, the identification of errors is based on source code analysis. Additionally, a set of test cases specifying the expected behaviour of the program is required - this set encompasses concrete values for variables, assertions, reachability constraints for paths, etc. An overview on different applications of model-based diagnosis techniques in software debugging is given in [13]. The representation of the intended behaviour of recommender user interfaces in the form of finite state representations is discussed in $[2,4]$. This approach is novel in the context of developing knowledge-based applications and, due to its formal foundation, allows an automated translation of the graphical representation into a recommender application. Basically there are three approaches to the implementation of recommender applications. Collaborative Filtering [6,9,12] and Content-based Filtering [9] do not exploit deep knowledge about the product domain. Collaborative Filtering is based on the assumption that customer preferences are correlated, i.e. similar products are recommended to customers with similar interest profiles. Content-based filtering focuses on the analysis of a given set of products already ordered by a customer. Based on this information, products are recommended which resemble products already ordered (products related to similar categories). Additionally, there are a number of approaches combining these basic approaches in order to gain an improved quality of the resulting solutions. Using knowledge-based approaches, the relationship between customer goals and preferences and offered products can be modelled explicitly. An overview on the Koba4MS development environment can be found in [4]. Such model-based knowledge representations are the major precondition for the application of model-based diagnosis [10] discussed in this paper.

\section{Conclusions}

In this paper we have presented an approach to the automated debugging of recommender process definitions representing the intended behaviour of user interfaces of knowledge-based recommenders. Predicate-based finite state representations are ap- 
plied in order to model interaction paths of recommender applications. For debugging purposes those finite state automata are translated into a corresponding representation of a constraint satisfaction problem. Results of an experiment show that our approach can significantly increase the productivity of recommender development.

\section{References}

[1] Burke, R. Knowledge-based Recommender Systems. Encyclopedia of Library \& Information Systems, 69, 32, 2000.

[2] Felfernig, A., and Shchekotykhin, K. Debugging User Interface Descriptions of Knowledge-based Recommenders. Workshop Notes of the IJCAI'05 Workshop on Configuration, 13-18, 2005.

[3] Felfernig, A., Friedrich, G., Jannach, D., and Stumptner, M. Consistency-based Diagnosis of Configuration Knowledge Bases. AI Journal 2, 152, 213-234, 2004.

[4] Felfernig, A. Koba4MS: Selling Complex Products and Services Using Knowledge-Based Recommender Technologies. $7^{\text {th }}$ IEEE International Conference on E-Commerce Technology, 92-100, 2005.

[5] Friedrich, G., Stumptner, M., and Wotawa, F. Model-based diagnosis of hardware designs. AI Journal 111, 2, 3-39, 1999.

[6] Herlocker, J. L., Konstan, J. A., Terveen, L. G., and Riedl, J. T. Evaluating Collaborative Filtering Recommender Systems. ACM Transactions on Information Systems 22, 1, 5-53, 2004.

[7] Hopcroft, J., and Ullman, J. Introduction to Automata Theory, Languages, and Computation. Addison-Wesley Publishing Company, Massachusetts, USA, 1979.

[8] Mateis C., Stumptner, M., and Wotawa, F. Modeling Java programs for diagnosis. 14 ${ }^{\text {th }}$ European Conference on Artificial Intelligence, 171-175, 2000.

[9] Pazzani, M. A Framework for Collaborative, Content- Based and Demographic Filtering. Artificial Intelligence Review 13, 5-6, 393-408, 1999.

[10] Reiter, R. 1987. A theory of diagnosis from first principles. AI Journal 23, 1, 57 95, 1987.

[11] Sachenbacher, M., Struss, P., and Carlen, C. A Prototype for Model-Based OnBoard Diagnosis of Automotive Systems. AICOM 13, 2, 83-97, 2000.

[12] Sarwar, B., Karypis, G., Konstan, J. A., and Riedl, J. T. Item-based collaborative filtering recommendation algorithms. 10 ${ }^{\text {th }}$ Int. WWW Conference, 285-295, 2001.

[13] Stumptner, M., and Wotawa, F. A Survey of Intelligent Debugging. AI Communications $11,1,35-51,1998$.

[14] Tsang, E. Foundations of Constraint Satisfaction. Academic Press, London, 1993.

[15] V.Noord, G., and Gerdemann, D. Finite State Transducers with Predicates and Identities. Grammars 4, 3, 263-286, 2001. 